\title{
An Optimization Model for Large Scale Airspace
}

\author{
Tolebi Sailauov and Z. W. Zhong
}

\begin{abstract}
South-East Asia is considered to be one of the fastest traffic growing regions in the world. Due to congested air traffic or bad weather conditions accidents happened in the history. This paper proposes a new mathematical model that finds optimal routes by taking into account delay cost, controller's capacity, departure and arrival capacities of an airport, waypoints capacity, routes capacity, rerouting options by using actual flight paths and considering "fairness" to aircrafts as well. The model can be applied to large scale airspace like ASEAN airspace.
\end{abstract}

Index terms-Mathematical model, objective function, constraints, airspace.

\section{INTRODUCTION}

Air traffic controllers' main purpose is to prevent collisions, organize traffic movements and give ground support to pilots. Controllers use traffic collision avoidance schemes like traffic separation rules, which makes sure that there is at least minimum separation between flights in all directions, which can be done using radar information. In addition, aircraft have collision avoidance systems, which further enhances safety by warning pilots when separation rules have been breached.

However, even with these technologies air traffic congestion occurs as a consequence of small weather disturbances. In order to deal with congestion there is a need for a system which can find optimal solution in a short time. That is why this paper proposes the mathematical model which can find optimal routes for a large scale environment. The model takes care of routes capacity, airport capacity, waypoints capacity and speed control of the aircraft. Also, the model allows rerouting options. With all these benefits it can be applied to the ASEAN airspace, which is one of the fastest air traffic growing regions in the world.

There is a need for an automatic system which can help controllers to find optimal solutions for congested airspace in reasonable time. That is why this paper proposes a mathematical model which can be used to solve congested problems in large scale.

The model to find optimal routes for the flights in large scale has been developed. The model considers airport constrains, waypoint constrains, and route constrains, safety, controller's workload, and gives rerouting options. It has an objective function which consists of airborne holding and ground holding cost which needs to be minimized.

Manuscript received March 11, 2016; revised April 30, 2016.

The authors are with the School of Mechanical and Aerospace Engineering School of Mechanical and Aerospace Engineering, Nanyang Technological University, Singapore (e-mail: Tolebi002@e.ntu.edu.sg, Mzwzhong@ntu.edu.sg).

\section{LITERATURE REVIEW}

There have been 2 approaches in general: optimizing airspace or optimizing routes. Good example for optimizing airspace can be [1], where they use spatio-temporal analysis to optimize airspace.

In the paper [2] the authors compare 3 methods: mixed-integer linear programming (MILP), MILP with first come first serve (FCFS) based heuristics and MILP with heuristics of genetic algorithms (GA). Durations of the computation and average delays of the eight flights are calculated in seconds and shown in Table I. Some of the durations of the computations with very long computational time were indicated as "N/A". MILP can find the optimal solution but it is very computationally costly. When we deal with a high amount of traffic it is not applicable. On the other hand MILP with GA has faster computation and still at optimal range. MILP with FCFS is somewhere in between MILP and MILP with GA.

The sum of total travel time has been chosen as an objective function for this paper. Also, the paper considers uncertainty (linear, quadratic) during flights and adds buffers.

TABLE I: COMPUTATION TIMES (IN SECONDS) TO SCHEDULE DIFFERENT NUMBER OF AIRCRAFTS [2]

\begin{tabular}{lcccccc}
\hline Number of flights & 6 & 7 & 8 & 40 & 100 & Average delay (8 flights), \\
\hline MILP,s & 6.71 & 98.3 & 1258 & N/A & N/A & 8.23 \\
MILP with FCFS-based heuristics, s & 2.10 & 33.2 & 419 & 2040 & N/A & 8.38 \\
MILP with heuristics of genetic algorithms, s & $<1.00$ & 1.00 & 2.20 & 23.4 & 50.0 & 8.58 \\
\hline
\end{tabular}

However this model has limitations. Firstly, the objective function seems to need some improvements. It does not consider cost, safety and fairness to the aircraft. Secondly, for all four cases during simulations all possible routes have been considered for each flight, which is very inefficient and that is probably why it cannot compute for a large amount of flights. Thirdly, by considering uncertainty and adding buffers, the result becomes much more astray from the optimal solution and loses the purpose of finding optimal solution.

Whenever one flight deviates from its schedule it affects other flights as well. For instance, in Europe about 24\% of all flights and in US about $18 \%$ of the all flights were delayed for more than 15 min [3]. Paper [4] suggests its own modelling in order to fast recover from congested system. The model was done graphically and mathematically. In this model airports are placed row by row. Lines show timeline and nodes show position of aircraft at that particular time.

Objective function:

$$
\sum_{f \in F} \sum_{k \in C C_{f}} c c_{f}^{k} a_{m}^{k}+\sum_{f \in F} c c_{f} b_{f}+\sum_{p m \in P M} \widetilde{c c}_{p m} n_{p m} \tau_{p m}
$$

The objective function consists of 3 parts: 
The first part is a flight plan cost where cost parameter $c c_{f}^{k}$ is a sum of the aircraft swap cost, flight delay cost and incremental fuel cost.

The second part is a cancellation cost.

The third part is a passenger misconnection cost, where $\widetilde{C C_{p m}}$ is an approximate re-accommodation cost for the passengers.

However this model has limitations as well. Model does not consider trajectories during flights. In the model flight is represented as a connection from one airport to another, so does not consider uniqueness of routes. It does not consider changing of routes and assumes the cost for delay, fuel cost are all constant for specific flight. For example, single value $c c_{f}^{k}$ is a sum of the aircraft swap cost, flight delay cost and incremental fuel cost. However, fuel cost, flight delay cost may vary depending on which route has been chosen, also, on weather conditions and where and how long delay was. It does not have safety, airspace and runway capacity limitations.

Another paper proposes integer programming (IP) model which should work for large scale airspace [5]. whereas:

$$
w_{j, t}^{f}=\left\{\begin{array}{c}
1, \text { if a flight } f \text { reaches sector } j \text { by time } t \\
0, \text { in other cases }
\end{array}\right.
$$

The objective function [4]:

$$
\begin{aligned}
& \operatorname{Min} \sum_{f \in F}\left(\sum_{t \in T_{\text {dest }_{f}}^{f}} c_{t d}^{f}(t)\left(w_{\text {dest }_{f}, t}^{f}-w_{\text {dest }_{f}, t-1}^{f}\right)\right. \\
&\left.-\sum_{t \in T_{\text {orig }_{f}}^{f}} c_{g}^{f}(t)\left(w_{\text {orig }_{f}, t}^{f}-w_{\text {orig }_{f}, t-1}^{f}\right)\right)
\end{aligned}
$$

The objective function is constructed in such a way that it first takes into account cost of the total delay then subtracts the reduction of the cost done by ground holding.

Constraints [1]:

$$
\sum_{f \in F: \text { orig }_{f}=k}\left(w_{k, t}^{f}-w_{k, t-1}^{f}\right) \leq \operatorname{Dep}_{k}(t) \quad \forall k \in \mathcal{R}, t \in \mathbb{T}
$$

$$
\sum_{f \in F: \text { dest }_{f}=k}\left(w_{k, t}^{f}-\mathrm{w}_{\mathrm{k}, \mathrm{t}-1}^{\mathrm{f}}\right) \leq \operatorname{Ar}_{\mathrm{k}}(\mathrm{t}) \quad \forall \mathrm{k} \in \mathcal{R}, \mathrm{t} \in \mathbb{T}
$$

$$
\sum_{f \in F: \mathrm{j} \in \mathcal{P}^{\mathrm{f}}}\left(\max \left\{0, w_{j, t}^{f}-\sum_{j^{\prime} \in \mathcal{L}_{\mathrm{j}}^{\mathrm{f}}} w_{j^{\prime}, t}^{f}\right\}\right) \leq \operatorname{Sec}_{j}(t) \forall j \in \mathcal{P}, \mathrm{t}
$$

$$
\begin{aligned}
& w_{j, t}^{f} \leq \sum_{j^{\prime} \in \mathcal{P}_{j}^{f}} w_{j^{\prime}, t-l_{f j j^{\prime} j}}^{f} \forall f \in F, t \in T_{j}^{f}, j \in \mathcal{P}^{\mathrm{f}}: j \\
& \quad \neq \operatorname{orig}_{\mathrm{f}} \\
& w_{j, \bar{T}_{j}^{f}}^{f} \leq \sum_{j^{\prime} \in \mathcal{L}_{j}^{f}} w_{j^{\prime}, \bar{T}_{j^{\prime}}^{f}}^{f} \quad \forall f \in F, j \in \mathcal{P}^{f}: j \neq \text { dest }_{f}
\end{aligned}
$$

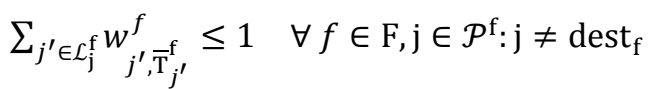

$$
\begin{aligned}
& \mathrm{w}_{\text {orig }}^{\mathrm{f}, \mathrm{t}}-\mathrm{w}_{\text {dest }_{\mathrm{f}^{\prime}}, \mathrm{t}-\mathrm{s}_{\mathrm{f}}}^{\mathrm{f}^{\prime}} \leq 0 \forall \mathrm{f}, \mathrm{f}^{\prime} \in \mathrm{F} \text { that are continued, } \mathrm{t} \\
& \in \mathrm{T}_{\text {orig }_{f}}^{\mathrm{f}}: \mathrm{t}-\mathrm{s}_{\mathrm{f}} \in \mathrm{T}_{\text {orig }}^{f^{\prime}} \\
& w_{\text {orig }_{f}, t}^{f}-w_{\text {dest }_{f}, t+e n d_{f}}^{f} \leq 0 \quad \forall f \in F, t \in T_{\text {orig }_{f}}^{f} \\
& : t+\text { end }_{f} \\
& \in T_{\text {dest }_{f}}^{f} \\
& w_{j, t-1}^{f}-w_{j, t}^{f} \leq 0 \quad \forall f \in F, t \in T_{j}^{f}, j \in \mathcal{P}^{f} \\
& w_{j, t}^{f} \in\{0,1\} \quad \forall f \in F, t \in T_{j}^{f}, j \in \mathcal{P}^{f}
\end{aligned}
$$

Constraint (2.1) ensures that number of flights departing at time $t$ in airport $k$ will not exceed departure capacity of the airport $k$ at time $t$. Constraint (2.2) ensures that number of flights arriving at time $\mathrm{t}$ to airport $\mathrm{k}$ will not exceed arrival capacity of the airport $\mathrm{k}$ at time t. Constraint (2.3) ensures that number of flights in sector $j$ at time $t$ will not exceed capacity of the sector $\mathrm{j}$ at time. Constraint (2.4) ensures that any flight f must spend at least $l_{f j^{\prime} j}$ units of time in preceding sector $j^{\prime}$ before reaching sector $\mathrm{j}$. Constraint (2.5) ensures that flight $\mathrm{f}$ must reach one of the subsequent sectors by latest time interval. Constraint (2.6) ensures that any flight will take only 1 path to flight. Constraint (2.7) ensures that if a flight has a stop at some airport then it must spend minimum required time $\mathrm{s}_{\mathrm{f}}$ at that stop. Constraint (2.8) ensures that any flight duration will not exceed maximum flight duration of that flight. It will ensure that none of the aircraft will get delayed for a very long time.Finally, Constraints (2.9) ensure connectivity in time.

Limitations

- There is no consideration of rerouting options inside sectors.

- Does not consider controllers workload.

- Cost for ground and airborne holding may differ in different sectors and airports.

- Takes sectors as routes instead of actual flight paths.

\section{Research Methodology}

\section{A. Limitations of Existing Studies}

From literature review it can be seen that all models mentioned above do not consider actual flight paths. That is why constrains on segments between waypoints, waypoints constrains cannot be addressed. Furthermore, controllers' workload and safety issue have not been considered. More importantly, there is no existing model which can find optimal solutions in reasonable time for large scale congested airspace which includes all airport capacities, route capacities and controller's workload. In addition, ground holding cost and airborne holding cost may differ depending on airport and airspace where an aircraft is. This was not taken into account.

This work for the first time proposes a new mathematical model, which can be used for large scale airspace, like the ASEAN airspace. The model considers all constrains mentioned above and gives a more realistic optimal solution for congested airspace. The model considers real flight paths, safety issues, and controller's workload as well, which are not included in other models. It takes into account differences 
in ground holding cost in different airports, and differences in airborne holding cost in different airspace. Airspace has many fixes and paths inside that have their own capacity, and this model takes care of these fix and path capacities. The new model takes into consideration airport, detailed airspace capacity, and more importantly controller's capacities as well. By having maximum durations of the flights we ensure "fairness" of the delays, so that none of the flights gets delayed for a very long time.

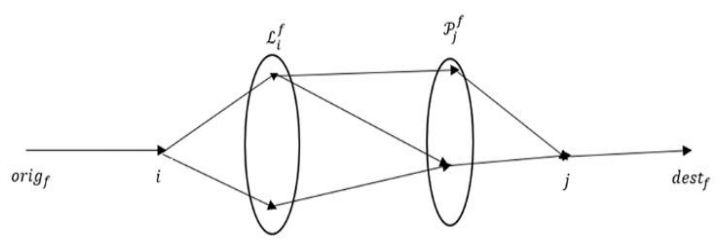

Fig. 1. Graphical representation of the simple flight.

\section{B. Mathematical Model}

A mathematical model can be represented mathematically and graphically. In this model all the routes are represented as a directed graph. Each node represents a waypoint (fix) or airport which can be flown by particular flight f. Edge shows route and direction of the flight. Figure1 shows an example of 1 flight.

$$
\text { Let } \mathrm{w}_{\mathrm{j}, \mathrm{t}}^{\mathrm{f}}=\left\{\begin{array}{c}
1, \text { if flight } \mathrm{f} \text { arrives at fix } \mathrm{j} \text { by time } \mathrm{t} \\
0 \text {, otherwise }
\end{array}\right.
$$

$\mathcal{R}$ - set of airports,

$\mathcal{P}$ - set of fixes,

$\mathcal{P}^{\mathrm{f}} \in \mathcal{P}$ - set of fixes that can be flown by flight $\mathrm{f}$, including the departure and arrival airports of flight $\mathrm{f}$

$F$ - set of all existing flights,

$\mathbb{T}$ - set of time intervals,

$\mathcal{L}_{\mathrm{i}}^{\mathrm{f}}-$ set of fixes that follow fix $\mathrm{i}$,

$\mathcal{P}_{\mathrm{j}}^{\mathrm{f}}-$ set of fixes that precede fix $\mathrm{j}$,

$\operatorname{Dep}_{k}(t)$ - departure capacity of airport k at time t,

$A r_{k}(t)$ - arrival capacity of airport k at time $\mathrm{t}$,

$S_{j}(t)$ - capacity of fix j at time $t$,

$\operatorname{dep}_{f}$ - anticipated take off time of flight $\mathrm{f}$,

$a r_{f}$ - anticipated landing time of flight $\mathrm{f}$,

$t_{f}$ - turnaround time of an airplane after flight $\mathrm{f}$,

$l_{f j j^{\prime}}$ - minimum number of time units that flight $\mathrm{f}$

must spend between $\mathrm{j}$ and $\mathrm{j}^{\prime}$

end $_{\mathrm{f}}-$ maximum allowed duration of flight $\mathrm{f}$

$$
T_{j}^{f}-\left[\underline{T}_{j}^{f}, \bar{T}_{j}^{f}\right]
$$

- set of feasible time intervals for flight $\mathrm{f}$ to land to fix $\mathrm{j}, T_{-}^{f}$ - first time interval in set $T_{j}^{f}$,

$\bar{T}_{j}^{f}$ - last time interval in set $T_{j}^{f}$.

Then the objective function will be:

$$
\begin{array}{r}
\operatorname{Min} \sum_{f \in F}\left(\sum_{t \in T_{\text {orig } f}^{f}} f_{G H}^{\text {orig }_{f}}\left(t-\operatorname{dep}_{f}\right)\left(w_{\text {orig }_{f}, t}^{f}-w_{\text {orig }_{f}, t-1}^{f}\right)\right. \\
\left.+\sum_{j \in \mathcal{P} f} \sum_{t \in T_{j}^{f}} f_{A H}^{j}\left(t-a r_{f, j}\right)\left(w_{j, t}^{f}-w_{j, t-1}^{f}\right)\right)
\end{array}
$$

where $\mathcal{P}^{\mathrm{f}}$ is a set of fixes that flight $\mathrm{f}$ can fly, $a r_{f, j}$ is a scheduled arrival time to fix $j$ of the flight $f . f_{G H}^{\text {orig }_{f}}(t)$ and $f_{A H}^{j}(t)$ are ground holding and airborne holding cost function for particular airport $\operatorname{orig}_{\mathrm{f}}$ and fix $\mathrm{j}$ such that:

$$
\begin{gathered}
f_{G H}^{\text {orig }_{f}}(t)-f_{G H}^{\text {orig }_{f}}(t-1) \geq 0, \\
f_{A H}^{j}(t)-f_{A H}^{j}(t-1) \geq 0 \forall t \in T_{j}^{f}
\end{gathered}
$$

Constraints:

$$
\sum_{f \in F}\left(w_{j, t}^{f}-w_{j, t-1}^{f}\right) \leq k_{j, t} \forall j \in \mathcal{P}
$$

$\sum_{f \in F: \text { orig }_{f}=a}\left(w_{k, t}^{f}-w_{k, t-1}^{f}\right) \leq \operatorname{Dep}_{k}(t) \forall a \in \mathcal{R}, t \in \mathbb{T}$ (3.2)

$\sum_{f \in F: \operatorname{dest}_{\mathrm{f}}=a}\left(w_{a, t}^{f}-w_{a, t-1}^{f}\right) \leq A r_{a}(t) \quad \forall a \in \mathcal{R}, \mathrm{t} \in \mathbb{T}$ (3.3)

$\sum_{f \in F: \mathrm{j} \in \mathcal{P}} \mathrm{f}\left(\max \left\{0, w_{j, t}^{f}-\sum_{j^{\prime} \in \mathcal{L}_{\mathrm{j}}^{\mathrm{f}}} w_{j^{\prime}, t}^{f}\right\}\right) \leq S_{j}(t) \forall j \in \mathcal{P}$,

$$
\mathrm{t} \in \mathbb{T}
$$

$w_{j, t}^{f} \leq \sum_{j^{\prime} \in \mathcal{P}_{j}^{f}}^{f} w_{j^{\prime}, t-l_{f j^{\prime} j}}^{f} \forall f \in F, t \in T_{j}^{f}, j \in \mathcal{P}^{f}: j \neq$ orig $_{f}$

$$
\mathrm{w}_{\mathrm{j}, \overline{\mathrm{T}}_{\mathrm{j}}^{\mathrm{f}}}^{\mathrm{f}} \leq \sum_{\mathrm{j}^{\prime} \in \mathcal{L}_{\mathrm{j}}^{\mathrm{f}}} \mathrm{W}_{\mathrm{j}^{\prime}, \overline{\mathrm{T}}_{\mathrm{j}^{\prime}}^{\mathrm{f}}}^{\mathrm{f}} \quad \forall \mathrm{f} \in \mathrm{F}, \mathrm{j} \in \mathcal{P}^{\mathrm{f}}: \mathrm{j} \neq \text { dest }_{\mathrm{f}}
$$

$\sum_{j^{\prime} \in \mathcal{L}_{\mathrm{j}}^{\mathrm{f}}} w_{j^{\prime}, \overline{\mathrm{T}}_{j^{\prime}}^{\mathrm{f}}}^{f} \leq 1$ for $\forall f \in \mathrm{F}, \mathrm{j} \in \mathcal{P}^{\mathrm{f}}: \mathrm{j} \neq$ dest $_{\mathrm{f}}$

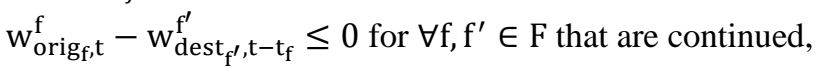

$$
\begin{gathered}
\mathrm{t} \in \mathrm{T}_{\text {orig }_{f}}^{\mathrm{f}}: \mathrm{t}-\mathrm{t}_{\mathrm{f}} \in \mathrm{T}_{\text {orig }_{f^{\prime}}}^{f^{\prime}} \\
w_{\text {orig }_{f}, t}^{f}-w_{\text {dest }_{f}, t+\text { end }_{f}}^{f} \leq 0 \text { for } \forall f \in F, t \in T_{\text {orig }_{f}}^{f} \\
t+\text { end }_{f} \in T_{\text {dest }_{f}}^{f} \\
w_{j, t-1}^{f}-w_{j, t}^{f} \leq 0 \text { for } \forall f \in F, t \in T_{j}^{f}, j \in \mathcal{P}^{f} \\
w_{j, t}^{f} \in\{0,1\} \text { for } \forall f \in F, t \in T_{j}^{f}, j \in \mathcal{P}^{f}
\end{gathered}
$$

Constraints (3.1) will ensure that the total number of aircraft will not exceed the capacity of that fix at time t. This constraint applicable for choke fixes, or congested fixes. Controllers can handle a particular amount of aircraft at time $t$ and at choke fix j. Constraint (3.2) ensures that the number of flights departing at time $\mathrm{t}$ in airport $a$ will not exceed take off capacity of the airport $\mathrm{k}$ at time t. Constraint (3.3) ensures that the number of flights arriving at time $\mathrm{t}$ to airport $a$ will not exceed landing capacity of the airport $a$ at time t. Constraint (3.4) ensure that the total number of flights that may fly between fix $\mathrm{j}$ and following fix $j^{\prime}$ at time $\mathrm{t}$ will not exceed the capacity of the segment between $\mathrm{j}$ and $j^{\prime}$ at time $\mathrm{t}$. It will ensure the capacity of that segment as well as safety of flights.

Constraint (3.5) ensures that any flight $f$ cannot reach fix $j$ on its path until it has spent at least $l_{f j^{\prime} j}$ time units traveling from one of the preceding fixes on its way. Constraint (3.6) ensures that flight $f$ must reach one of the subsequent fixes by latest time period. Constraint (3.7) ensures that any flight will take only 1 path to flight. Constraint (3.8) ensures that if a flight has a stop at some airport then it must spend minimum required time $s_{f}$ at that stop. Constraint (3.9) ensures that any flight duration will not exceed maximum flight duration of that flight. It also ensures "fairness" of the delays so that none of the flights gets delayed for very long time. Finally, constraints (3.10) and (3.11) come from definition of the w.

Advantages of the new model:

The model uses actual routes instead of flown sectors or 
airports like in papers reviewed in Section 2. The model enables more detailed and more accurate representation of

routes in real life.

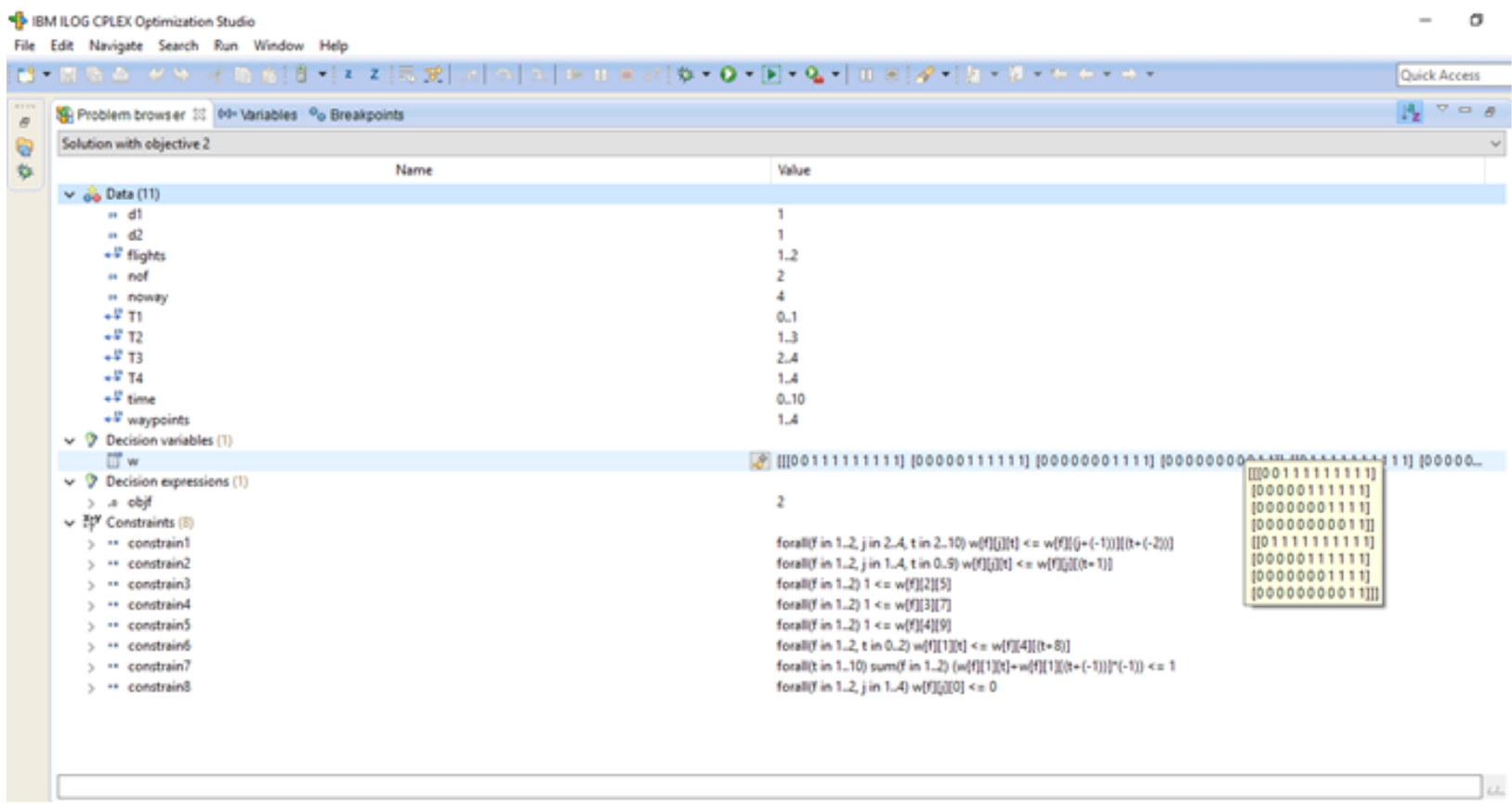

Fig. 2. Screenshot of the results from CPLEX software.

- The objective function consists of superposition of ground holding and airborne holding delay costs. Linear superposition of the objective function will ensure fast calculation of optimal routes.

- It takes into account differences in ground holding cost in different airports, and differences in airborne holding cost in different airspace.

- $\quad$ Airspace has many fixes and paths inside that have its own capacities, and this model takes care of the fix and path capacities. Fix capacities are determined by how many flights a controller can handle at that fix and at time t. Path capacity is determined by flight levels, minimum separation between flights, and length of the path.

- $\quad$ There is only one variable $w_{j, t}^{f}$ which can take value 0 or 1 which must satisfy all constraints. Once the values for $w_{j, t}^{f}$ have been determined, the objective function value will be determined. The $w_{j, t}^{f}$ values which satisfy these constraints and the minimum value of the objective function will be the solution to this problem.

- It takes into consideration airport, detailed airspace capacity (fixes and paths capacity), and more importantly controller's capacities.

- By having the maximum duration of the flight we ensure "fairness" of the delays, so that none of the flights gets delayed for a very long time.

\section{CASE Study}

A case study was conducted in order to show that the new model is working. In the case study 2 flights have been used. Both of them are supposed to flight from airport 1 to airport 4 through fixes 3 and 4 (1-2-3-4). The scheduled departure time is 1 for both flights. The objective function consists of only ground holding costs. Ground holding delay functions are
$f_{G H}^{\text {orig }_{f_{1}}}(t)=2 t^{2}$ and $f_{G H}^{\text {orig }_{f_{2}}}(t)=3 t$.

- Constraint 1 ensures that any flight should spend at least 2 units of time from one fix to another.

- Constraint 2 ensures connectivity of the path. If a flight has reached any fix by time $t$ then it must reach the subsequent fix by time $t+1$.

- Constraints 3-5 ensure that a flight $f$ must reach one of the subsequent fixes by the latest time interval at which it is allowed to reach these fixes.

- Constraint 6 ensures that the total flight time will not overstep the maximum tolerable duration of the flight.

- Constraint 7 ensures departure capacities of the airports have been met.

- Constraint 8 is used for initialization purposes.

The simulation results show that the new model works. The model minimized the objective function to 2 . The departure capacity was equal to 1 . It delayed flight 1 for 1 unit of time. See Fig. 2 for more details.

\section{CONCLUSION}

As we know, human can make mistakes, and air traffic controllers are not an exception. The new model has been proposed to help air traffic flow management teams to solve congested airspace problems on a large scale. The main advantage of the model is that it uses actual routes, which is why it is able to consider ground holding constraints, airborne holding constraints, airport capacities, waypoint capacities, route capacities and controllers' capacity. It gives a "fair" solution, which means that none of the aircraft will be delayed for a very long time. The objective function is constructed in such a way that it takes into account differences in ground delay cost and airborne delay cost in different airspace and airports. Also, a case study has been conducted on CPLEX to show the efficiency of the new model. In the future, the model can be applied to any 
countries' airspace or ASEAN airspace.

\section{REFERENCES}

[1] J. Nosedal et al., "An optimization model to fit airspace demand considering a spatio-temporal analysis of airspace capacity," Transportation Research Part C: Emerging Technologies, vol. 61, pp. $11-28,2015$

[2] S. Choi et al., "Design of an optimal route structure using heuristics-based stochastic schedulers," Journal of Aircraft, 2015. vol. 52, no. 3, pp. 764-777.

[3] F. Eurocontrol, U.S./Europe Comparison of 2010 ATM-Related Operational Performance. [Online]. Available: http://www.faa.gov/air_traffic/publications/media/us_eu_comparison 2010.pdf

[4] B. Vaaben and J. Larsen, "Mitigation of airspace congestion impact on airline networks," Journal of Air Transport Management, vol. 47, pp. $54-65,2015$.
[5] D. Bertsimas, G. Lulli, and A. Odoni, "An integer optimization approach to large-scale air traffic flow management," Institute for Operations Research and the Management Sciences, p. 211, 2011

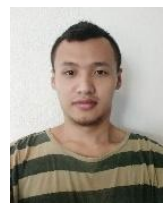

Tolebi Sailauov received his bachelor of engineering degree in electrical and electronical engineering from Nanyang Technological University, Singapore in 2014. He is currently pursuing his Ph.D. degree in mechanical and aerospace engineering, Nanyang Technological University, Singapore. His research interests include optimization of traffic in airspace, forecasting of the air traffic.

Z. W. Zhong worked at the Institute of Physical and Chemical Research, Japan after he received his doctor of engineering in precision engineering. He has also worked at the Gintic Institute of Manufacturing Technology, Singapore, and is currently at the Nanyang Technological University, Singapore. His research and development areas are precision engineering, air traffic management, mechatronics and design. 